\title{
MCL-1 plays an oncogenic role in breast cancer by modulating chemoresistance and stemness properties by activating Wnt/ $\beta$-catenin
}

\author{
Yuzhu Zhang \\ Ling Zhu \\ Guangdong Provical Hospital of Chinese Medicine \\ Yanmei Zhang \\ Guangdong provicial hospital of Chinese Medicine \\ Hai Lu \\ Guangzhou University of Chinese Medicine

\section{Xiaoqing Wei} \\ Guangzhou University of Chinese Medicine \\ Rui Xu \\ Guangdong Provical hospital of Chinese medicine \\ Liping Ren \\ Guangdong Provicial Hospital Chinese Medicine \\ Qianjun Chen ( $\nabla$ cqj55@163.com) \\ Guangdong Provicial of Chinese medicine
}

Guangdong Provicial Hospital of Chinese Medicine https://orcid.org/0000-0002-7010-3537

Primary research

Keywords: MCL-1, Breast cancer, chemoresistance, stemness, Wnt/ $\beta$-catenin

Posted Date: July 22nd, 2020

DOI: https://doi.org/10.21203/rs.3.rs-46792/v1

License: (9) (7) This work is licensed under a Creative Commons Attribution 4.0 International License. Read Full License 


\begin{abstract}
Background络east cancer is the most common malignant tumor and the leading cause of death in women. Chemotherapy is one of the most important treatments for breast cancer. However, the development of chemotherapy resistance is the main cause of $20-30 \%$ of breast cancer patients developing metastasis, leading to death. MCL-1, an anti-apoptotic protein, has not been found to contribute to chemotherapy resistance in breast cancer.
\end{abstract}

Methods $₫$ We used large gene panels to detect pathological sections of tumors in drug-resistant and sensitive patients. We validated protein profiling by IHC in a larger cohort of samples. We performed the function of MCL-1 by knockdown and overexpression in vitro and in vivo. Luciferase assay and CHIP assay were used to prove the regulatory network between MCL-1 and LRP6.

Result:We found that MCL-1 is more highly expressed in drug-resistant breast cancer tissues than it is in sensitive breast cancer tissues. Functional studies have revealed that MCL-1 plays an important role in drug resistance by regulating apoptosis in breast cancer cells. We found that overexpression of $\mathrm{MCL}-1$ enhances the chemoresistance and stemness of breast cancer cells in vitro and in vivo, while silencing has the opposite effect. Mechanistically, by downregulating and upregulating MCL-1, we show that MCL-1 regulates LRP6 and activates the WNT/ $\beta$-catenin signaling pathway in breast cancer cells. Finally, we found that a high level of MCL-1 expression predicts a poor prognosis in breast cancer.

Conclusion:Our work highlights the role of MCL-1 in chemoresistance and stemness. The MCL-1-WNT/ $\beta$-catenin axis might be used as a new clinical target for breast cancer therapy.

\title{
1. Background
}

Breast cancer is the most common malignant tumor in women, and it is the leading cancer-related cause of death in women. In $2018,2.1$ million women worldwide suffered from breast cancer, and one in every four female cancer patients had breast cancer [1]. Chemotherapy is one of the most important treatments for breast cancer [2]. However, because of chemotherapy resistance, 20-30\% of breast cancers will recur and metastasize, leading to death [3]. According to WHO data, there are currently 627,000 women worldwide who die of breast cancer [1]. In China, approximately 69,000 women die each year from breast cancer [4]. It is well known that the recurrence and metastasis of breast cancer after chemotherapy is essentially caused by the residual resistance of breast cancer cells to chemotherapy. Therefore, it is of great significance to study the chemoresistance mechanism of breast cancer and explore how to reduce chemoresistance and increase chemosensitivity to prevent breast cancer recurrence and metastasis and reduce the risk of breast cancer death. Breast cancer was also confirmed to involve cells with stemness characteristics [5]. These cells with stem cell characteristics are responsible for tumor growth and drug resistance $[6,7]$.

MCL-1 encodes an anti-apoptotic protein that is an important member of the Bcl-2 family [8]. Multiple transcript variants can be produced as a result of alternative splicing, and they have many anti-apoptotic roles $[9,10]$. MCL-1 protein localizes in the outer mitochondrial membrane, sequestering Bak and preventing the release of cytochrome $\mathrm{c}[11,12]$. A recent study showed that targeting MCL-1 by either treatment an inhibitor or an siRNA sensitizes gastric, colorectal and pancreatic cancer cells $[13,14]$. For breast cancer, a variety of studies have shown that there is an identified relationship between MCL-1 and resistance $[15,16]$. However, interestingly, we found that MYC may cooperate with MCL-1 to maintain cancer stem cells (CSCs) [5].

However, we found that the copy number of MCL-1 in chemotherapy resistant breast cancer patients was higher than it was in sensitive patients, as shown by Foundation One CDx assays [17]. In our previous studies, we established a breast cancer cell line resistant to multiple drugs (including Taxol), MDA-MB231/MDR (231/MDR), which has properties of cancer stem cells [18]. Herein, we demonstrate that MCL-1 is overexpressed in 231/MDR cells. Thus, we wondered whether MCL-1 independently functions to enhance the characteristics of stem cells in breast cancer.

\section{Materials And Methods}

\subsection{Cell lines and culture conditions}

The breast cancer cell lines MDA-MB-231 and MCF-7 were obtained from the American Type Culture Collection (ATCC; Manassas, VA). These breast cancer

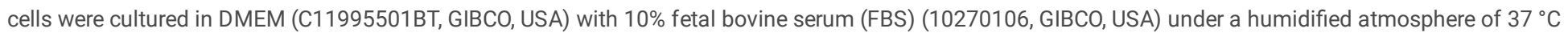
and $5 \% \mathrm{CO}_{2}$ (WCl-180, Wiggens, Germany). The multidrug-resistant cell line MDA-MB-231/MDR was induced by cisplatin (MB1055, Dalian Mellon, China) by treating with a range of low to high concentrations [18].

\subsection{Immunofluorescence}

Cells were seeded in 6-well plates and incubated overnight to allow the cells to attach, after which they were treated with DMSO (D2650, Sigma, USA) or taxol (5 nM) (MB1178, Dalian Mellon, China) with or without MCL-1 inhibitor (M-i) (S63845, MCE) for $48 \mathrm{~h}$. Then, cells were washed with PBS, fixed with acetone for 10 min at room temperature, permeabilized with $0.3 \%$ Triton X-100 in PBS for 30 min at $37^{\circ} \mathrm{C}$, blocked with $5 \%$ BSA in PBS for 30 min, and incubated with anti-MCL-1 (1:100, CST, Boston, USA) and anti-CD44 (1:100, CST, Boston, USA) overnight at $4{ }^{\circ} \mathrm{C}$. After that, the cells were incubated with goat anti-mouse 488 (ab150113, Abcam, England) and goat anti-rabbit 555 (ab150078, Abcam, England) for $1 \mathrm{~h}$ at room temperature. Cells were mounted with DAPI and observed under a laser confocal microscope (LSM710,ZEISS $₫$ Germany).

\subsection{Western blot analysis}

Cells were homogenized in RIPA buffer supplemented with protease inhibitors (CW22005, Kangwei, Beijing, China), and the protein concentrations were detected using a BCA assay kit (CWBIO, Beijing, China). After normalizing to obtain an equal protein concentration, samples were resuspended in SDS sample Loading [MathJax]/jax/output/CommonHTML/jax.js proteins were transferred onto polyvinylidene difluoride membranes (ISEQ00010, Millipore, SUA). Then, 
membranes were blocked with $5 \%$ BSA and probed at $4{ }^{\circ} \mathrm{C}$ overnight using the following antibodies: anti-MCL- 1 antibody, anti-CD 44 antibody (1:1000), antiOCT4 antibody (1:1000, CST, Boston, USA), anti-NANOG antibody (1:1000, CST, Boston, USA), anti-ALDHA1 antibody (1:1000, CST, Boston, USA) and antiGAPDH antibody (1:1000, CST, Boston, USA). After incubation with the appropriate secondary antibody for $1.5 \mathrm{~h}$ at room temperature, proteins were visualized by enhanced chemiluminescent reagent on a multifunctional imaging system (Tanon 5200).

\subsection{MCL-1 overexpression and inhibitor}

MB231 and MCF-7 cell lines were stably transfected with a lentiviral vector (pReceiver-Lv105-pure vector) by lentiviral transduction (MOI = 3), and they were selected by treatment with $5 \mathrm{\mu g} / \mathrm{ml}$ puromycin (219453925, MPbio, USA). Human MCL-1 cDNA (GeneCopoeia, EX-Z9207-Lv105-B, cDNA clone) or an empty vector was seeded into the pReceiver-Lv105-pure vector and were selected by treatment $2 \mu \mathrm{g} / \mathrm{ml}$ puromycin. MCL-1 was analyzed by WB and qRT-PCR. The MCL-1 inhibitor (M-i) S63845 was purchased from MCE. We used it at $10 \mathrm{~nm}$, which is a concentration that results in no cytotoxicity.

\subsection{Cell viability assay}

Cells (5000 cells/well) were seeded in 96 -well plates and placed in the cell culture incubator at $37^{\circ} \mathrm{C}$ overnight to allow the cells to attach, after which they were treated with taxol at concentrations of $0,1.625,3.125,6.250,12.500,25.000,50.000,100.000,200.000 \mathrm{nM}$ in the presence or absence of $\mathrm{M}-\mathrm{i}(5 \mathrm{~nm}$ in

DMSO) for 48 h. Cell viability was determined by MTT ((3-(4,5-dimethylthiazol-2-yl)-2,5-diphenyl tetrazolium bromide) assay (Sigma-Aldrich, Saint Louis, MO, USA), and then the percentages of viable cells was measured by absorbance value (OD) (VICTOR X5, PerkinElmer, USA) at $490 \mathrm{~nm}$. The experiments were repeated three times.

\subsection{Mammosphere formation assay}

Cells were grown in serum-free DMEM supplemented with 1 X B27 and were plated in ultralow attachment plates at a density of 10,000 viable cells/mL, and then they were grown for 10 days. Mammospheres were observed under a microscope and were photographed. The experiments were repeated three times.

\subsection{Colony formation assay}

NC and MCL-1-overexpressing cells were seeded into a 24-well plate $\left(5 \times 10^{2}\right.$ cells/well) and cultured in DMEM for $48 \mathrm{~h}$. Then, the fixed colonies formed were stained with crystal violet. The colony formation assay was repeated three times.

\subsection{Flow cytometry}

CD44 and CD24 were analyzed using flow cytometry. NC and MCL-1-overexpressing cells were then suspended in flow cytometry staining buffer at a final cell concentration of $1 \times 10^{6}$ cells $/ \mathrm{mL}$. Cells were incubated with the following fluorescent monoclonal antibodies (eBioscienceTM, Thermo Fisher Scientific): CD44 and CD24. Cells were washed two times with $2 \mathrm{~mL}$ of flow cytometry staining flow cytometry buffer and resuspended in $500 \mu \mathrm{L}$ of flow cytometry staining buffer. Flow cytometry analyses were performed on a BD flow cytometer (Calibur, BD, USA). The experiment was performed three times.

\subsection{RNA extraction and real-time PCR}

RNA was extracted from cells using TRIzol reagent (Takara, Beijing, China), and total RNA was reverse transcribed into cDNA. Quantitative real-time PCR was carried out using TB Green ${ }^{\text {TM }}$ Premix Ex Taq ${ }^{\text {TM }}$ II (Takara, Beijing, China) with a CFX96 ${ }^{\text {TM }}$ Real-Time System (ABI Quant Studio 7 Flex, Applied Biosystems, USA). GAPDH was used as the control, and the relative mRNA levels were analyzed by the $\left.2^{(-\triangle \triangle C t}\right)$ method. The primer sequences are listed in Table 1 . 
Table 1

Real-Time PCR primers for genes

\begin{tabular}{|c|c|c|}
\hline Gene & \multicolumn{2}{|c|}{ Primers Squence $\left(5^{\prime}->3^{\prime}\right)$} \\
\hline \multirow[t]{2}{*}{ MCL-1 } & Forward & ATGCTTCGGAAACTGGACAT \\
\hline & Reverse & TCCTGATGCCACCTTCTTCTAGG \\
\hline \multirow[t]{2}{*}{ BCL-1 } & Forward & TCTGCGAGGAACAGAAGTGC \\
\hline & Reverse & GAAATCGTGCGGGGTCATTG \\
\hline \multirow[t]{2}{*}{$B C L-x L$} & Forward & TGGTGAGTCGGATTGCAAGTT \\
\hline & Reverse & GTCAGGAACCAGCGGTTGAA \\
\hline \multirow[t]{2}{*}{ BAX } & Forward & AGCTCTGAGCAGATCATGAAGAC \\
\hline & Reverse & AGTTGAAGTTGCCGTCAGAAAAC \\
\hline \multirow[t]{2}{*}{ BID } & Forward & ACAGGGTACGATAACCGGGA \\
\hline & Reverse & GGGCCGTACAGTTCCACAAA \\
\hline \multirow[t]{2}{*}{ BAD } & Forward & GTGACGAGTTGTGGACTCCT \\
\hline & Reverse & ATCCCACCAGGACTGGAAGA \\
\hline \multirow[t]{2}{*}{ CD44 } & Forward & СCCTGCTACCAGACACTCA \\
\hline & Reverse & TGTTCACCAAATGCACCAT \\
\hline \multirow[t]{2}{*}{ LRP6 } & Forward & TGCAGAATGTTGTTGTTTCTG \\
\hline & Reverse & AATTAGAAACTTCAATCCGATTA \\
\hline \multirow[t]{2}{*}{ sox2 } & Forward & TGCTGCCTCTTTAAGACTAGGAC \\
\hline & Reverse & СCTGGGGCTCAAACTTCTCT \\
\hline \multirow[t]{2}{*}{ OCT4 } & Forward & ССTGTCTCCGTСАССАСТСТ \\
\hline & Reverse & CAAAAACCCTGGCACAAACT \\
\hline \multirow[t]{2}{*}{ Nanog } & Forward & TTTGTGGGCCTGAAGAAAACT \\
\hline & Reverse & AGGGCTGTCCTGAATAAGCAG \\
\hline \multirow[t]{2}{*}{ GAPDH } & Forward & TGGTGAAGGTCGGTGTGAAC \\
\hline & Reverse & GCTCCTGGAAGATGGTGATGG \\
\hline
\end{tabular}

\subsection{Clinical information and tissue samples}

This study was approved by the Ethics Committee of Guangdong Hospital of Traditional Chinese Medicine. All patients provided written informed consent to participate in the study. A total of 168 breast cancer (Table 2) tissues from patients with neoadjuvant chemotherapy sensitivity (NAC-S), neoadjuvant chemotherapy resistance (NAC-R), post neoadjuvant chemotherapy (post-NAC) and pre-neoadjuvant chemotherapy (pre-NAC) were used for the detection of MCL-1 and CD44 by IHC. Clinical information included basic information, tumor subtype, Ki67 expression and regimens. The overall survival (OS) times were calculated from the date of surgery to the end of follow-up. 
Table 2

Correlation between MCL-1 expression and clinicopathologic characteristics of BC patients.

\begin{tabular}{|c|c|c|c|c|c|}
\hline \multirow[t]{2}{*}{ Characteristic } & \multicolumn{2}{|c|}{$N A C-R(N=41)$} & \multicolumn{2}{|c|}{$N A C-S(N=127)$} & \multirow[t]{2}{*}{$P$} \\
\hline & $\mathbf{N}$ & $\%$ & $\mathbf{N}$ & $\%$ & \\
\hline \multicolumn{6}{|c|}{ Age at diagnosis, years } \\
\hline Mean \pm SD & \multicolumn{2}{|c|}{$51.15 \pm 12.02$} & \multicolumn{2}{|c|}{$50.87 \pm 8.95$} & 0.874 \\
\hline \multicolumn{6}{|l|}{ Median(range) } \\
\hline$\leq 44$ & 12 & 29.3 & 25 & 19.7 & \multirow[t]{3}{*}{0.045} \\
\hline $45-60$ & 20 & 48.8 & 88 & 69.2 & \\
\hline$\geq 61$ & 9 & 22.0 & 14 & 11.0 & \\
\hline \multicolumn{6}{|l|}{ Subtype } \\
\hline Luminal & 22 & 53.7 & 47 & 37.0 & \multirow[t]{4}{*}{0.069} \\
\hline HER2(+) & 12 & 29.3 & 53 & 41.7 & \\
\hline TNBC & 7 & 17.1 & 25 & 19.7 & \\
\hline NA(not available) & 0 & 0.0 & 2 & 1.6 & \\
\hline \multicolumn{6}{|l|}{ Ki 67} \\
\hline$\bigotimes 50 \%$ & 37 & 90.2 & 108 & 85.0 & \multirow[t]{2}{*}{0.254} \\
\hline$\geq 50 \%$ & 4 & 9.8 & 19 & 15.0 & \\
\hline \multicolumn{6}{|l|}{ Regimens } \\
\hline Taxol-based & 37 & 90.2 & 124 & 97.6 & \multirow[t]{2}{*}{0.06} \\
\hline Others & 4 & 9.8 & 3 & 2.4 & \\
\hline
\end{tabular}

\subsection{Animals}

All animal experiments were ethically carried out and were approved by the animal experiment committee of Guangdong Hospital of Traditional Chinese Medicine. Six-week-old female nude mice were purchased from Guangdong Medical Laboratory Animal Center (Fushan, Guangdong, China), and they were housed and fed in standard pathogen-free conditions. For tumor-limiting dilution assays, $1 \times 10^{3}, 5 \times 10^{3}, 1 \times 10^{4}, 5 \times 10^{4}$ and $1 \times 10^{5} \mathrm{MDA}-\mathrm{MB}-231$ cells with or without MCL-1 overexpression were mixed with Matrigrl matrix (1:1, BD Biosciences, San Jose, CA, USA) and orthotopically implanted in the inguinal mammary gland of mice. Stem cell frequencies were calculated using an ELDA (http://bioinf.wehi.edu.au/software/elda/). Additionally, $1 \times 10^{5}$ MDA-MB-231 cells were implanted in the inguinal mammary gland of mice. Ten days later, when the tumors reached a volume of $100 \mathrm{~mm}^{3}$, we randomly allocated the mice to groups in which they received taxol ( $5 \mathrm{mg} / \mathrm{kg})$, MCL- 1 with taxol $(5 \mathrm{mg} / \mathrm{kg}), \mathrm{M}-\mathrm{i}(2.5 \mathrm{mg} / \mathrm{kg})$ with taxol $(5 \mathrm{mg} / \mathrm{kg})$ or DMSO. Mice were euthanized $35 \mathrm{days}$ after the administration.

\subsection{Immunohistochemistry}

Immunohistochemistry (IHC) was conducted on paraffin-embedded sections. Before staining, paraffin sections were incubated at $60{ }^{\circ} \mathrm{C}$ for $1 \mathrm{~h}$ for dewaxing and treatment with dimethylbenzene two times for $15 \mathrm{~min}$. The tissue sections were soaked in a series of ethanol solutions with concentrations of $100 \%$, $100 \%, 95 \%, 80 \%$ and $75 \%$ for $5 \mathrm{~min}$ and then soaked in distilled water for $5 \mathrm{~min}$. Afterwards, the tissue sections were incubated with sodium citrate antigen retrieval solution for $10 \mathrm{~min}$ and in $3 \% \mathrm{H} 2 \mathrm{O} 2$ for $15 \mathrm{~min}$, which was followed by $5 \% \mathrm{BSA}$ for $40 \mathrm{~min}$ at $37^{\circ} \mathrm{C}$. Then, the tissue sections were incubated overnight at $4{ }^{\circ} \mathrm{C}$ with a primary anti-MCL-1 antibody (1:200 dilution in PBS, CST, Boston, USA) or an anti-CD44 antibody (1:200 dilution in PBS, CST, Boston, USA) and goat anti-mouse or anti-rabbit IgG for $30 \mathrm{~min}$ at room temperature. MCL-1 expression was visualized by DAB staining, and tissue sections were counterstained with hematoxylin. Finally, tissue sections were evaluated under light microscopy (Olympus BX53, Olympus, Japan).

\subsection{Transcriptional reporter gene assay}

Cells overexpressing MCL-1 and control cells were cultured in 96-well plates at a concentration of 5000 cells per well. Two hundred nanograms of TCF reporter plasmid, GLI reporter plasmid or RBP-JK reporter plasmid (Millipore) and $10 \mathrm{ng}$ of pRL-TK (Renilla-TK-luciferase vector, Promega) were cotransfected into the cells. After 48 h, a Dual-Glo Luciferase reporter Assay System (E1910, Promega) was used to measure the luciferase activities following the manufacturer's instructions.

\subsection{Coimmunoprecipitation assay and coculture immunoprecipitation assay}

For the coimmunoprecipitation assay, MB-231 cell lysates transfected with Flag-tagged MCL-1 or a vector control were subjected to immunoprecipitation with Flag antibody (1:1000) or control IgG for $2 \mathrm{~h}$ at $4^{\circ} \mathrm{C}$. All immunoprecipitations were performed with protein A/G sepharose (Santa Cruz Biotechnology) while 
rotating at $4{ }^{\circ} \mathrm{C}$ overnight. The beads were collected by centrifugation at $3000 \mathrm{rpm}$ and then were washed three times with lysis buffer. The immunoprecipitants were subjected to WB.

For the coculture IP assay, 293T cells were cultured in 6-well plates and individually transfected with $0.5 \mu$ g of MCL-1-Flag expression plasmids or LRP6-HA, Wnt3a-myc or Wnt10b-His plasmids (Thermo Fisher Scientific, Shanghai) using Lipofectamine 3000. After $24 \mathrm{~h}$ of coculture, cell lysates with LRP6 receptors and cell lysates with Wnt3a or Wnt10b ligands were prepared at a ratio of 1:2:2 to detect the interaction of the LRP6 receptor and two ligands. The subsequent steps were the same as the IP assay mentioned above.

\subsection{Statistical analysis}

Data are presented as the means \pm standard deviation, and GraphPad Prism (version 8, San Diego, CA, USA) was used to conduct statistical analyses. The significance of a difference between the groups was tested using Student's $t$-test for the data. A $P$-value $<0.05$ was considered statistically significant.

\section{Results}

\subsection{MCL-1 is associated with resistance to taxol in breast cancer patients}

Three breast cancer patients, one with resistance to chemotherapy and the others with sensitivity, were tested with Foundation One CDx assays. The copy number of MCL-1 in Taxol-resistant breast cancer patients was 32, which was much higher than that in sensitive breast cancer patients (Supplementary Excel 1). Overexpression of MCL-1 has been reported to be associated with poor prognosis in cancers [19]. MCL-1 is a member of the Bcl-2 family. To investigate the role of the Bcl-2 protein family in drug-resistant patients, we performed real-time qRT-PCR to analyze the expression of $M C L-1, B c l-1, B c l-x L, B A X, B I D$ and $B A D$ in the multichemotherapy-resistant in the MDA-MB-231/MDR (231/MDR) cell line and the sensitive cell line. Real-time RT-PCR analysis showed that the expression of MCL-1 was significantly higher in 231/MDR cells than it was in sensitive cells (Fig. 1a). Furthermore, we examined the expression of MCL-1 in the tumors of 168 patients for neoadjuvant therapy by using IHC. IHC staining revealed that MCL- 1 was remarkably elevated in neoadjuvant chemotherapy resistance (NAC-R) compared to that of neoadjuvant chemotherapy sensitivity (NAC-S) (Fig. 1b, c and d). Furthermore, to confirm that chemotherapy drug resistance could upregulate MCL-1 expression, we detected MCL-1 expression by ICH before and after neoadjuvant chemotherapy. We randomly chose 20 of the 168 patients to measure MCL-1 expression in the puncture tissue samples before neoadjuvant therapy and after. The results showed that MCL- 1 expression in the tumor tissues significantly increased after receiving neoadjuvant therapy (Fig. 1e, $\mathrm{f}$ and g). Collectively, our results reveal that MCL- 1 is upregulated in chemoresistant breast cancer. In addition, we also confirmed by immunofluorescent analysis that the protein level of MCL-1 was strongly upregulated in breast cancer mammospheres (Fig. 1h) derived from 2 independent patients. Similarly, western blot analysis revealed that compared to control cells, the mammospheres exhibited higher expression of Nanog, OCT4 and SOX2, which are transcription factors involved in the maintenance of stem cell properties $[20,21]$.

\subsection{Chemotherapies promote MCL-1 overexpression in breast cancer cell lines.}

To investigate the effect of chemotherapy drugs on MCL-1 expression in breast cancer, we used western blotting to analyze MCL-1 expression in the multidrugresistant cell line 231/MDR and in wild-type MDA-MB-231 cells (231). The results showed that MCL-1 expression in 231/MDR cells was much higher than it was in 231 cells (Fig. 2b). Interestingly, we found that 231/MDR had a stronger ability to induce cancer stem cell colony formation (Fig. 2a) and higher expression of Nanog, OCT4 and SOX2 (Fig. 2b). Meanwhile, real-time RT-PCR and western blotting were used to analyze MCL-1 expression in MB-213 and MCF-7 cells treated with different concentrations of taxol ( $1 \mathrm{nM}, 5 \mathrm{nM}$, and $10 \mathrm{nM})$, which is the most common drug in chemotherapy regimens. Real-time RTPCR and western blot results revealed that cells treated with higher taxol concentrations had higher levels of MCL-1 mRNA and protein (Fig. 2c and d). All the results above demonstrate MCL-1 overexpression in breast cancer cell lines after chemotherapy.

\subsection{MCL-1 depresses sensitivity to taxol in breast cancer cell lines to regulate stem cell transformation.}

To elucidate the mechanism of MCL-1 in taxol resistance, we overexpressed MCL-1 in two breast cancer cell lines, MDA-MB-231 (MB231) and MCF-7, using a lentivirus-based expression system. The western blot results showed significant overexpression of MCL-1 in cells transfected with MCL-1-expressing lentivirus compared with the control vector (Fig. 3a). To confirm the anti-apoptotic effect of MCL-1 on breast cancer cells, we conducted apoptosis analysis, cell viability assays and MTT assays. MTT assay results also confirmed that MCL- 1 could inhibit taxol-induced cell death. MTT assays showed that the IC ${ }_{50}$ values of MCL-1-overexpressing cells were dramatically increased compared with those of the control vector (Fig. $3 \mathrm{~b}$ and c). Similar findings were observed in flow cytometry results, which showed that overexpression of MCL-1 had an anti-apoptotic effect in MB231 and MCF-7 cells treated with different concentrations of taxol (1, 5 or $10 \mathrm{nM})$ (Fig. 3d).

We hypothesized that MCL-1 would induce cellular drug resistance by increasing breast cancer cell stemness. To test this hypothesis, we first conducted a mammosphere formation assay to investigate the relationship between MCL-1 expression and mammosphere formation ability. The results show that MCL-1 overexpression dramatically increased mammosphere formation by MB231 and MCF-7 cells in number and diameter (Fig. 3e-f). Then, we used flow cytometry to test the expression of CD 44 and CD24. $C D 44^{+} / C D 24^{-}$cells in breast tumors have cancer stem cell characteristics [22].

In addition, we examined the mRNA and protein expression of CD44 and three other typical stemness cell markers, OCT4, NANOG and SOX2. They were all upregulated in both MB231 and MCF-7 cells transfected with MCL-1 vectors, as assessed by RT-PCR and western blot (Fig. 4a b). CD44 is a glycoprotein that resides at the cell surface. MCL-1 is located at the outer mitochondrial membrane and mitochondrial matrix in pluripotent stemness cells [23].

Loading [MathJax]/jax/output/CommonHTML/jax.js

Page 6/18 
Immunofluorescence staining revealed that the expression of MCL-1 (green) correlated with CD44 (red) in cells (Fig. 4c). We have proven that the expression level of MCL-1 in resistant 231/MDR cells is much higher than it is in MB-231 cells (Fig. 1a). To comprehensively clarify the effect of MCL-1 on taxol resistance, we blocked MCL-1 expression in MDR cells by treating them with the MCL-1 inhibitor M-i. MTT assays revealed that MDR-231 cells treated with the MCL-1 inhibitor M-i regained sensitivity to taxol (Fig. 5a, b). As expected, the mammosphere forming ability of the MCL-1 blocking cells was weakened (Fig. 5c). The expression levels of SOX2, Nanog, OCT4 and CD44 were decreased with the inhibition of MCL-1 by western blot and immunofluorescence (Fig. $5 \mathrm{~d}$, e), suggesting that cancer cell stems were eliminated. Taken together, these data demonstrated that overexpression of MCL-1 is greatly increased in cancer stem cells and depresses taxol sensitivity in breast cancer cells.

\subsection{MCL-1 activates the WNT/ $\beta$-catenin pathway}

Having shown the effects of MCL-1 on chemotherapy resistance and cancer stem cells. Next, we investigated the signaling pathway by which MCL- 1 alters the function of cancer stem cells. WNT/ $\beta$-catenin, Notch and Hedgehog have been reported to play essential roles in cancer stem cells [24, 25]. Herein, we examined which signaling pathway is involved in MCL-1 in breast cancer by a dual-luciferase reporter gene assay. The MB231 cells overexpressing Mcl-1 and MCF-7 cells were transfected into Renilla and one of the following signal pathway plasmids: TCF/catenin plasmid (WNT/ $\beta$-catenin reporter plasmid), RBP plasmid (Notch reporter plasmid) or GLI plasmid (Hedgehog reporter plasmid). The results showed that WNT/ $\beta$-catenin signaling was significantly activated, while the other signaling pathways experienced no significant change (Fig. 6a-c). We further confirmed that WNT/ $\beta$-catenin signaling is inhibited by silencing MCL-1 (Fig. $6 \mathrm{~d}$ ). Staining of $\beta$-catenin showed that nuclear localization of $\beta$-catenin in MCL-1-overexpressing cells was more evident than it was in control cells, as shown by immunofluorescence data (Fig. 6e). Western blotting also revealed similar results, showing that $\beta$-catenin was increased in MCL-1overexpressing cells (Fig. 6f). To further clarify that MCL-1 activates WNT/ $\beta$-catenin signaling in breast cancer, small molecule inhibitors of WNT/ $\beta$-catenin (CWP232228; HY-18959 MCE) [26], Notch inhibitor 1 (HY-12860 MCE) and Hedgehog inhibitor (CUR61414; HY-113965 MCE) were used for our experiments. We treated MB231 and MCF-7 cells with $5 \mathrm{nM}$ concentrations of the inhibitors and different concentrations of taxol at the same time. The data showed that upregulated MCL-1 increased drug resistance and that the WNT/ $\beta$-catenin inhibitor reversed it (Table 3 ). These results have shown that MCL-1 activates the WNT/ $\beta$-catenin signaling pathway and promotes the nuclear translocation of $\beta$-catenin. Blocking the WNT/ $\beta$-catenin signaling pathway reversed the effects of MCL-1 on breast cancer cell drug resistance.

Table 3 Half maximal inhibitory concentration $\left(\mathrm{IC}_{50}\right)$ of groups cells treated with Taxol (the mean $\pm \mathrm{SD}$ is from 3 independent experiments)

\begin{tabular}{|lll|}
\hline & Taxol $₫ \mathrm{nM} \rrbracket$ \\
\hline Vector & MCF-7 & MB-231 \\
\hline MCL-1 & $1.05 \pm 0.05$ & $2.45 \pm 0.11$ \\
\hline MCL-1+CWP232228 & $25.36 \pm 1.34$ & $21.49 \pm 1.08$ \\
\hline Vector+CWP232228 & $0.75 \pm 0.03$ & $1.56 \pm 0.08$ \\
\hline MCL-1+CUR61414 & $27.71 \pm 1.89$ & $19.48 \pm 2.01$ \\
\hline Vector+ CUR61414 & $1.64 \pm 0.09$ & $1.78 \pm 0.12$ \\
\hline MCL-1+ Notch inhibitor 1 & $19.78 \pm 1.27$ & $20.91 \pm 2.75$ \\
\hline Vector+ Notch inhibitor 1 & $0.33 \pm 0.02$ & $0.75 \pm 0.04$ \\
\hline MCL-1+siLRP6 & $5.26 \pm 0.23$ & $4.75 \pm 0.17$ \\
\hline Vector+siLRP6 & $1.13 \pm 0.03$ & $1.78 \pm 0.06$ \\
\hline
\end{tabular}

Next, to investigate how MCL-1 activates the WNT/ $\beta$-catenin signaling pathway, we used coimmunoprecipitation (Co-IP) to examine the interaction between MCL-1 and WNT proteins. The results showed that MCL-1 had no interaction with the breast cancer WNT proteins wnt 3a, wnt 10b and Frizzld 6 (Fzd 6 ), but there was an interaction between MCL-1 and the WNT pathway receptor LRP6 (Fig. $6 \mathrm{~g}$ and $6 \mathrm{~h}$ ). To confirm the interaction, we silenced LRP6 and used different concentrations of taxol before analyzing the cells by MTT assay. The data showed that silencing LRP6 reversed taxol resistance (Table 3 ). Furthermore, we investigated by IP how MCL-1 modulates the WNT receptor interaction. Cocultured 293T cells were transfected with MCL-1-Flag, LRP6-HA, Wnt 3a-myc and Wnt 10b-His plasmids (Fig. 6i and 6j). Cell lysates were immunoprecipitated with an HA antibody and immunoblotted with anti-Flag, anti-HA, anti-Myc or anti-His antibodies. The results confirmed that LRP6 coimmunoprecipitated with MCL-1, Wnt 3a, Wnt10b and LRP6. Moreover, upregulated MCL-1 enhances the binding between Wnt 10b and LRP6. These results suggest that MCL-1 directly interacts with LRP6 and increases the binding between the WNT receptor and ligands in cells.

\subsection{MCL-1 overexpression is associated with tumorigenesis and taxol resistance in vivo.}

We have demonstrated that MCL-1 overexpression induces taxol resistance in vitro. Furthermore, we conducted tumorigenicity assays and xenograft tumor growth assays. Tumorigenicity assays revealed that the tumorigenicity of MCL-1-overexpressing cells was remarkably stronger than that of control cells $(P<$ 0.0001; Fig. 7a). Moreover, the overexpression MCL-1 xenograft nude mouse group had a poor prognosis (Fig. 7b). In the xenograft tumor growth assay, both the tum mo size and weinht were remarkahlv reduced in the MCL-1 inhibitor M-i + taxol group compared with the DMSO group, the MCL-1 overexpression + taxol Loading [MathJax]/jax/output/CommonHTML/jax.js

Page $7 / 18$ 
group and taxol group (Fig. 7c-g). Taken together, our data revealed that MCL-1 plays a very important role in taxol resistance and the characteristics of cancer stem cells in breast cancer.

\subsection{A high level of MCL-1 expression predicts chemoresistance in breast cancer.}

Identifying markers of breast cancer stem cells and chemoresistance is important for efficacy stratification in the clinic. To confirm the correlation between MCL-1 expression and poor prognosis in breast cancer patients, we classified the 168 breast cancer patients used in this study into 4 subtypes according to their MCL-1 staining intensity of and followed up. The patients with high MCL-1 expression had a poor prognosis compared with the patients with low MCL-1 expression ( $P<0.05$; Fig. 8a). To further clarify the correlation between MCL-1 and cancer stem cell relevant genes (CD44 and LRP6), we performed qRT-PCR on biopsy tissues from the 168 breast cancer patients. There was a significant positive correlation between MCL-1 and CD44 ( $\left.R^{2}=0.3603, P<0.0001\right)$ and LRP6 ( $\left.R^{2}=0.4356, P<0.0001\right)$ (Fig. 8b). These results implied that upregulated MCL-1 enhances chemoresistance by modulating stemness properties.

\section{Discussion}

Breast cancer is the most common malignant tumor in women and the leading cancer that threatens women's lives. It is well known that the recurrence and metastasis of breast cancer after chemotherapy is essentially caused by the residual resistance of breast cancer cells to chemotherapy. Therefore, it is of great significance to study the mechanism of chemoresistance in breast cancer, explore how to reduce chemoresistance and increase chemosensitivity to prevent recurrence and metastasis of breast cancer and reduce the risk of death of breast cancer. Here, we identified MCL- 1 as a novel signature gene of breast cancer stem cells. First, we found that MCL-1 gene expression is higher in NAC-R patients than it is in NAC-S patients and that MCL-1 expression is greatly increased in mammospheres compared to monolayers in primary 3D culture. Second, we used the multidrug resistance model 231/MDR to confirm the correlation of MCL-1 expression and taxol sensitivity. Third, overexpressing MCL-1 enhances mammosphere formation and upregulates the levels of stemness-associated genes SOX2, Nanog and OCT 4 in breast cancer cells. Fourth, we found that the expression of MCL- 1 was positively correlated with CD44 by immunohistochemical staining. Fifth, a high level of MCL-1 expression predicts poor prognosis in breast cancer.

MCL-1 encodes an anti-apoptotic protein that is a member of the Bcl-2 family [27]. Two research teams, those of Kelly, G. L and Gasca, J, reported that MCL-1 and other Bcl-2 family proteins were altered in response to chemotherapy [28-29]. MCL-1 and WNT/ $\beta$-catenin were both listed as potential therapeutic target genes in liver cancer[30]. In our study, we confirmed that MCL-1 activated WNT/ $\beta$-catenin signaling in breast cancer by following evidence. First, overexpressing and silencing MCL-1 promoted and reduced the activation of WNT/ $\beta$-catenin signaling, as shown by luciferase reporter assay. Second, the nuclear translocation of $\beta$-catenin was found by western blot and immunofluorescence staining assays to be increased following MCL-1 upregulation. Moreover, IP assays found that MCL-1 interacts with LRP6, and Co-IP assay discovered that MCL-1 may help LRP6 proteins interact with the WNT/ $\beta$-catenin ligand receptor Wnt 10b. Taken together, we conclude that MCL-1 plays an important role in the progression of breast cancer though the WNT/ $\beta$-catenin signaling pathway (Fig. 9).

The WNT/ $\beta$-catenin signaling pathway has been implicated in oncogenesis and in several developmental processes, including regulation of cell fate and patterning during embryogenesis [31]. This pathway supports stem and cancer stem cells [32]. When the concentration of $\beta$-catenin in the cytoplasm reaches a certain level, it can translocate to the nucleus and combine with TCF/lefs, which lead to cell proliferation, differentiation and maturation [33]. Several studies in different tumors have confirmed that the WNT/ $\beta$-catenin signaling pathway mediates stem cell renewal and drug resistance by promoting translocation to the nucleus of $\beta$-catenin [34-36]. Our data provide evidence that MCL-1 promotes cancer stem cell characteristics and drug resistance though the WNT/ $\beta$ catenin signaling pathway, which offers a novel strategy for targeted therapy in breast cancer.

Our research finds that MCL-1 is a stem cell signature gene in breast cancer and is a novel regulator of the WNT/ $\beta$-catenin signaling pathway. However, a limitation of our research is the number of samples and the retrospective analysis. Prospective, multicenter, large sample clinical trials are needed to further confirm the predictive value of MCL-1.

\section{Conclusion}

Our team determined a new role of MCL-1 in chemotherapy resistance and prognosis for breast cancer patients. We revealed that MCL-1 mediates cancer stemness cell and taxol resistance both in vitro and in vivo. The effect is associated with the activation of the WNT/ $\beta$-catenin signaling pathway, which directly interacted with LRP6 to regulate its expression. These findings provide evidence that the MCL-1-WNT/ $\beta$-catenin axis may be a target for breast cancer therapy, which offers novel strategies for the precise treatment of breast cancer.

\section{Abbreviations}

OS: Overall survival; PCR:Polymerase chain reaction; qRT-PCR:Quantitative reverse transcription-PCR; ICH:Immunohistochemistry; CHIP:chromatin immunoprecipitation assay; CSCs:cancer stem cells; ATCC:American Type Culture Collection; DMSO:Dimethyl sulfoxide; DMEM:Dulbecco's Modified Eagle Media; NC:control group; NAC-S:neoadjuvant chemotherapy sensitivity; NAC-R:neoadjuvant chemotherapy resistance.

\section{Declarations}

\section{Availability of data and materials}

Loading [MathJax]/jax/output/CommonHTML/jax.js plication and related data files. 


\section{Conflict of interest}

Authors declare no conflict of interest.

\section{Ethics approval and consent to participate}

The tissue samples used for the study were approved by the Ethics Committee of Guangdong Provincial Hospital of Chinese Medicine. The protocols for animal experiments were approved by the Institutional Animal Care and Use Committee and Institutional Biosafety Committee of Guangdong Provincial Hospital of Chinese Medicine.

\section{Consent for publication}

Not applicable.

\section{Funding}

This study was supported by grants from the National Natural Science Foundation of China (No. 81904206 and 81974571 ); Guangdong Natural Science Foundation (No. 2017A030313719; Foundation Project of Guangzhou University of Traditional Chinese Medicine ( No. XKP2019002).

\section{Acknowledgments}

Designed the experiments: Yuzhu Zhang and Qianjun Chen. Performed the experiments: Yuzhu Zhang, Ling Zhu, and Yanmei Zhang. Analyzed the data: Xiaoqing Wei and Hai Lu. Wrote the paper: Yuzhu Zhang. Provided clinical biological samples: Rui Xu and Liping Ren. All authors read and approved the final manuscript.

\section{References}

1. Bray F, Ferlay J, Soerjomataram, et al. Global cancer statistics 2018: GLOBOCAN estimates of incidence and mortality worldwide for 36 cancers in 185 countries[J]. CA Cancer J Clin. 2018;68(6):394-424.

2. NCCN Clinical Practice Guidelines in. Oncology (NCCN Guidelines $\left.{ }^{\circledR}\right)$, Breast Cancer, Version 3.2018, BINV-K 1 OF 5.

3. Cardoso F, Spence D, Mertz S, et al. Global analysis of advanced/metastatic breast cancer: Decade report (2005-2015) [J]. Breast (Edinburgh, Scotland), 2018, 39:131-138.

4. Chen W, Zheng R, Baade PD, et al. Cancer statistics in China, 2015[J]. CA Cancer J Clin. 2016;66(2):115-32.

5. Lee KM, Giltnane JM, Balko JM, et al. MYC and MCL1 Cooperatively Promote Chemotherapy-Resistant Breast Cancer Stem Cells via Regulation of Mitochondrial Oxidative Phosphorylation[J]. Cell Metab. 2017;26(4):633-47.e7.

6. Zhu R, Gires O, Zhu L, et al. TSPAN8 promotes cancer cell stemness via activation of sonic Hedgehog signaling[J]. Nat Commun. 2019;10:2863.

7. Perone Y, Farrugia A, Rodríguez Meira A, et al. Author Correction: SREBP1 drives keratin-80-dependent cytoskeletal changes and invasive behavior in endocrine-resistant ERa breast cancer[J]. Nat Commun. 2019;10:3791.

8. Huang K, O’Neill KL, Li J, et al. BH3-only proteins target BCL-xL/MCL-1, not BAX/BAK, to initiate apoptosis[J]. Cell research, $2019: 1-11$.

9. Han Y, Wu N, Jiang M, et al. Long non-coding RNA MYOSLID functions as a competing endogenous RNA to regulate MCL-1 expression by sponging miR29c-3p in gastric cancer[J]. Cell proliferation, 2019: e12678.

10. Wan Y, Li Y, Yan C, et al. Indole: A privileged scaffold for the design of anti-cancer agents[J]. European journal of medicinal chemistry, $2019: 111691$.

11. Goan YG, Wu WT, Liu Cl, et al. Involvement of Mitochondrial Dysfunction, Endoplasmic Reticulum Stress, and the PI3K/AKT/mTOR Pathway in NobiletinInduced Apoptosis of Human Bladder Cancer Cells[J]. Molecules. 2019;24(16):2881.

12. Milani M, Beckett AJ, Al-Zebeeby A, et al. DRP-1 functions independently of mitochondrial structural perturbations to facilitate BH3 mimetic-mediated apoptosis[J]. Cell death discovery. 2019;5(1):117.

13. Taniai M, Grambihler A, Higuchi H, Werneburg N, Bronk SF, Farrugia DJ, et al. Mcl-1 mediates tumor necrosis factor-related apoptosis-inducing ligand resistance in human cholangiocarcinoma cells. Cancer Res. 2004;64(10):3517-24.

14. Maeta Y, Tsujitani S, Matsumoto S, Yamaguchi K, Tatebe S, Kondo A, et al. Expression of Mcl-1 and p53 proteins predicts the survival of patients with T3 gastric carcinoma. Gastric Cancer. 2004;7(2):78-84.

15. Hu Y, Yagüe, Ernesto, Zhao J, et al. Sabutoclax, pan-active BCL-2 protein family antagonist, overcomes drug resistance and eliminates cancer stem cells in breast cancer[J]. Cancer Letters, 2018: S0304383518301770.

16. Louault K, Bonneaud TL, Séveno C, et al. Interactions between cancer-associated fibroblasts and tumor cells promote MCL-1 dependency in estrogen receptor-positive breast cancers[J]. Oncogene, 2019.

17. Wacheck V, Cejka D, Sieghart W, Losert D, Strommer S, Crevenna R, et al. Mcl-1 is a relevant molecular target for antisense oligonucleotide strategies in gastric cancer cells. Cancer Biol Ther. 2006;5(10):1348-54.

18. Yuzhu Zhang, Jingjing W, Meina Y, et al. ETS1 is associated with cisplatin resistance through IKKa/NF-KB pathway in cell line MDA-MB-231[J]. Cancer Cell Int. 2018;18(1):86-98.

Loading [MathJax]/jax/output/CommonHTML/jax.js 
19. Cronin M, Ross JS. Comprehensive next-generation cancer genome sequencing in the era of targeted therapy and personalized oncology[J]. BIOMARKERS IN MEDICINE, 2011, 5(3):293-305.

20. Takahashi K, Tanabe K, Ohnuki M, et al. Induction of Pluripotent Stem Cells From Adult Human Fibroblasts by Defined Factors[J]. Cell. 2007;131(5):86172.

21. Wu CW, Wang ML, Chiou SH. Targeting cancer stem cells: emerging role of Nanog transcription factor[J]. OncoTargets Therapy. $2013 ; 6: 1207$.

22. Hu Y, Smyth GK. ELDA: Extreme limiting dilution analysis for comparing depleted and enriched populations in stem cell and other assays. J Immunol Methods. 2009;347:70-8.

23. Campbell KJ, Dhayade S, Ferrari N, et al. MCL-1 is a prognostic indicator and drug target in breast cancer[J]. Cell death \& disease, $2018,9(2): 19$.

24. Fox RG, Park FD, Koechlein CS, et al. Musashi Signaling in Stem Cells and Cancer[J]. Annu Rev Cell Dev Biol. 2015;31(1):249-67.

25. Ouspenskaia T, Matos I, Mertz A, et al. WNT-SHH Antagonism Specifies and Expands Stem Cells prior to Niche Formation[J]. Cell. 2016;164(1-2):156-69.

26. Jang GB, Hong IS, Kim RJ, et al. Wnt//2-Catenin Small-Molecule Inhibitor CWP232228 Preferentially Inhibits the Growth of Breast Cancer Stem-like Cells[J]. Can Res. 2015;75(8):1691-702.

27. Wright MH, Calcagno AM, Salcido CD, et al. Brca1 breast tumors contain distinct CD44+/CD24- and CD133 + cells with cancer stem cell characteristics[J]. Breast Cancer Res. 2008;10(1):R10.

28. Rasmussen ML, Kline LA, Park KP, et al. A non-apoptotic function of MCL-1 in promoting pluripotency and modulating mitochondrial dynamics in stem cells[J]. Stem cell reports. 2018;10(3):684-92.

29. Kelly GL, Strasser A. The essential role of evasion from cell death in cancer.Adv. Cancer Res. 2011;111:39-96.

30. Comprehensive and Integrative Genomic Characterization of Hepatocellular. Carcinoma[J] Cell. 2017;169(7):1327-41.e23.

31. Zhang J, Zhou, Bin, Liu Y, et al. Wnt inhibitory factor-1 functions as a tumor suppressor through modulating Wnt/ $\beta$-catenin signaling in neuroblastoma[J]. Cancer Letters, 348(1-2):12-19.

32. Clevers H. Wnt/ $\beta$-Catenin Signaling in Development and Disease[J]. Cell. 2006;127(3):0-480.

33. Park H, Kim Y, Yu B, et al. Alternative Wnt Signaling Activates YAP/TAZ[J] Cell. 2015;162(4):780-94.

34. Lu Y, Xie S, Zhang W, et al. Twa1/Gid8 is a $\beta$-catenin nuclear retention factor in Wnt signaling and colorectal tumorigenesis[J]. Cell Res. 2017;27(12):1422-40.

35. Aggarwal A, Prinz-Wohlgenannt M, Gr?Schel C, et al. The calcium-sensing receptor suppresses epithelial-to-mesenchymal transition and stem cell- like phenotype in the colon[J]. Molecular Cancer. 2015;14(1):61.

36. Christian Schürch, Riether C, Matter MS, et al. CD27 signaling on chronic myelogenous leukemia stem cells activates Wnt target genes and promotes disease progression[J]. Journal of Clinical Investigation. 2012;122(2):624-38.

\section{Figures}




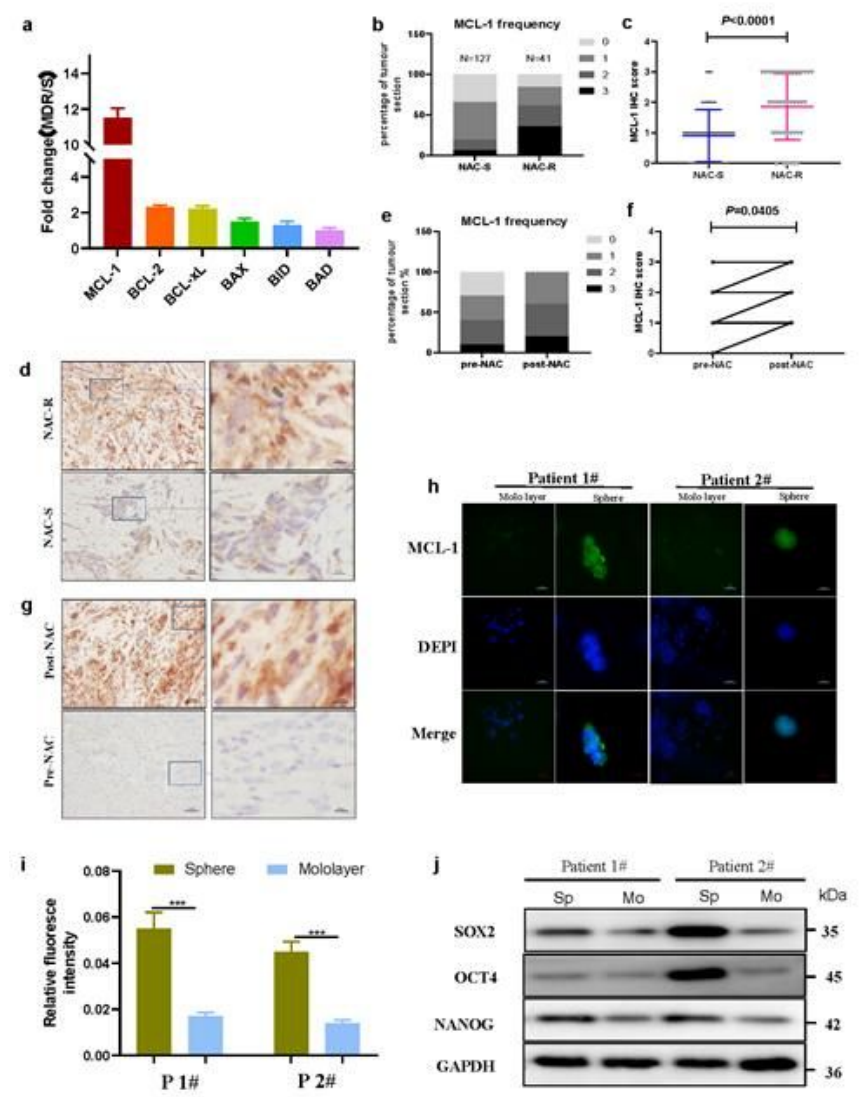

Figure 1

MCL-1 expression is significantly associated with drug resistance in breast cancer cells and tissue. (a) Bcl-2 family expression levels are detected in breast cancer drug resistant cell line 231/MDR compared to sensitive cell line MDA-MB-231 by qRT-PCR. Data are shown as fold change by 2 (- $\triangle \triangle \mathrm{Ct}$ ) method and the mean $\pm S D$ is from 3 independent experiments. (b-d) Immunohistochemistry analyses of MCL-1 expression in specimens of breast patients' tissues with neochemotherapy sensitivity (NAC-S) and resistance (NAC-R). According to the pathological scoring system and its representative MCL- 1 staining, our categorization is negative $=0$, low expression $=1$, medium expression $=2$, high expression $=3$ (scale bar=100\&25 $\mu \mathrm{m}, \mathrm{P} \otimes 0.0001, \mathrm{n}=168) .(\mathrm{e}-\mathrm{g}) \mathrm{MCL}-1$ expression in specimens of breast patients' tissues with before and after neo-chemotherapy (pre-NAC vs post-NAC) by ICH (scale bar=100\&25 $\mu \mathrm{m}$, $\mathrm{P}=0.0405$, $\mathrm{n}=20$ ), Note that some dots overlapped. (h) Freshly isolated primary human tissues derived from two independent breast cancer patients were grown as adherent cells or spheres. Representative fluorescence images for MCL-1 (green), and nuclei (blue) of adherent cells or spheres from two breast cancer

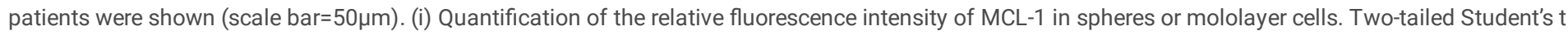
test was used for statistical analysis. ${ }^{\star *} \mathrm{P}<0.001,{ }^{*} \mathrm{P}<0.01$ and error bars represent standard deviation from three times of independent experiments. (j) Immunoblotting analyses of SOX2, OCT 4 and Nanog were performed between spheres and adherent cells. 
Figure 2
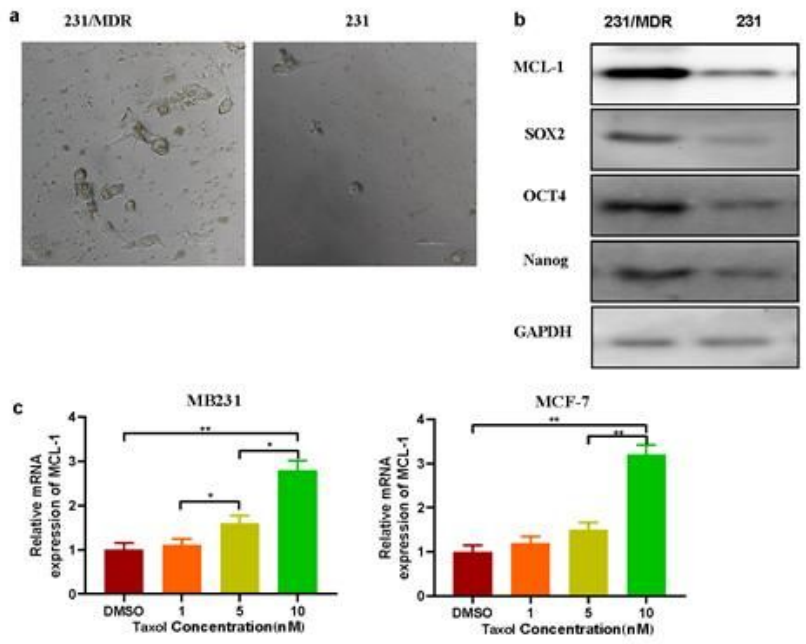

d

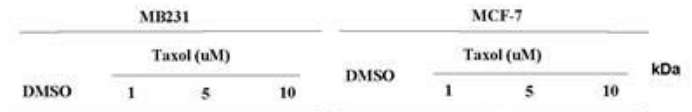

MCL-1

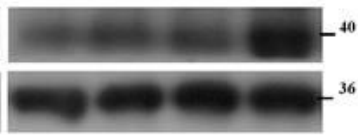

GAPDH

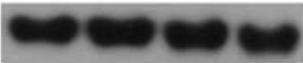

\section{Figure 2}

Chemotherapy drug promotes MCL-1 overexpression in breast cancer cell lines. (a) Tumor sphere forming ability of the indicated cells between drug resistant cell 231/MDR and sensitive cell 231 was examined. (b) Immunoblotting analyses ofMCL-1, SOX2, OCT4 and Nanog were performed between drug resistant cell 231/MDR and sensitive cell 231.(c-d) MCL-1 expression are evaluated by qRT-PCR and WB in breast cancer cell lines after intervention different dose of $\operatorname{Taxol}(0,1,5,10 \mu \mathrm{M})$ for $48 \mathrm{~h}$. Those figures above are representative results of 3 independently repeated experiments. ${ }^{*} P \llbracket 0.05 \nabla^{\star *} \mathrm{P} \otimes 0.01$ (student`s t-test) 
Figure 3
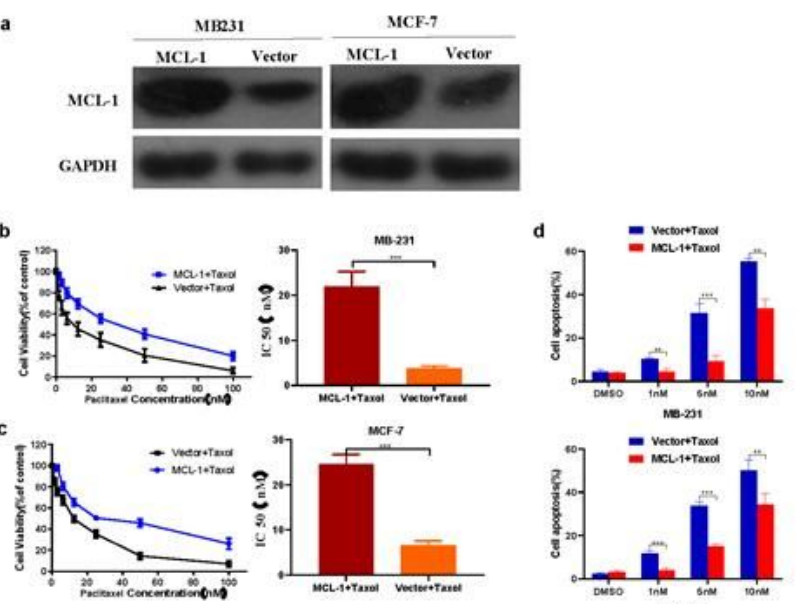

"1] MCF

- vectoretrad
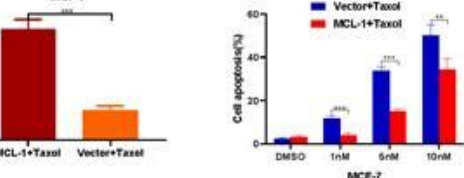

MCL-1
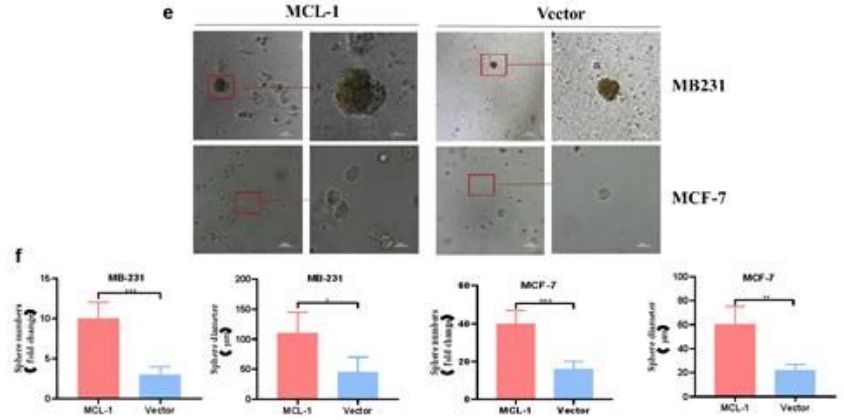

Figure 3

Overexpression MCL-1 depresses sensitivity to Taxol in breast cancer cell lines and regulates cancer stem cell transformation. MB231-MCL-1 (MCL-1), MB231vector (Vector), MCF-7-MCL-1(MCL-1) and MCF-7-vector (Vector) stable cells are tested by WB. (b-c) MTT assay shows that cells viability is significantly higher in MCL-1 overexpression with different concentrations of Taxol. MCL-1 overexpression groups

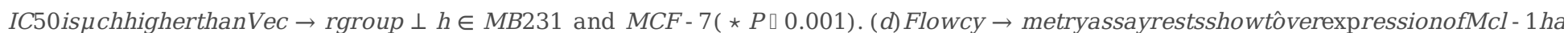

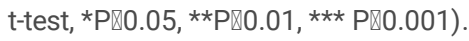


Figure 4

a
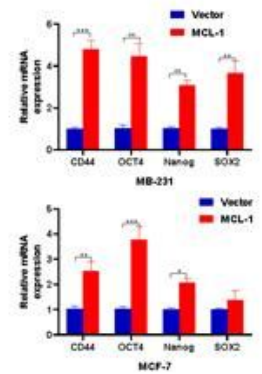

mat.

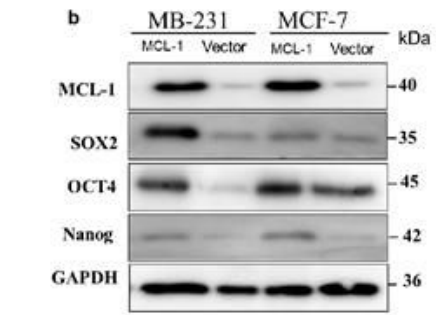

c

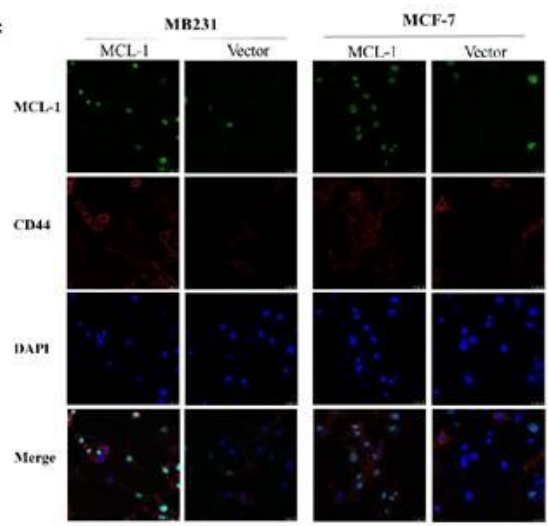

\section{Figure 4}

MCL-1 regulates the expression of stemness cell relevant genes in breast cancer. (a-b) Quantitative RT-PCR and WB results show CD44, SOX2 OCT4 and Nanog expression increased in MCL-1 upregulated group. (c): Immunofluorescence colocalization results show representative image for MCL-1 (green), CD44

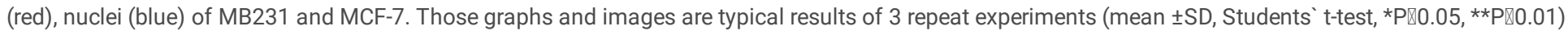




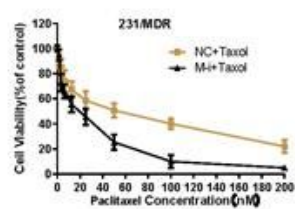

c
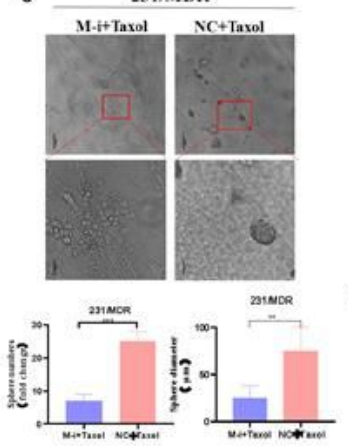

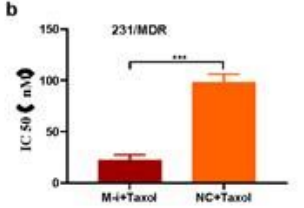

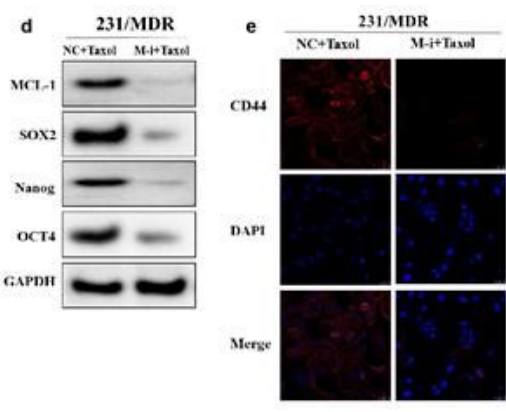

Figure 5

MCL-1 inhibitor M-i overcomes Taxol resistance and eliminates cancer stem cells in MDR breast cancer cells. (a) MTT assay shows the viability of breast cancer cell lines decreased in different concentration Taxol with M-i (5 nm in DMSO) and DMSO. (b) IC50 is much lower with M-i than NC in 231/MDR. (c) Histograms show the representative diameters and numbers of sphere formation MB231/MDR, which are cultured for 120 hours with M-i or not. (d-e) Intervention with M-i, the CD44, SOX2 OCT4, Nanog and MCL-1 expression levels are tested by WB and immunofluorescence. Those graphs and images are representative results of 3 repeat experiments (mean $\pm S D$, Students` $t$-test, ${ }^{* * * P \otimes 0.001) . ~}$ 


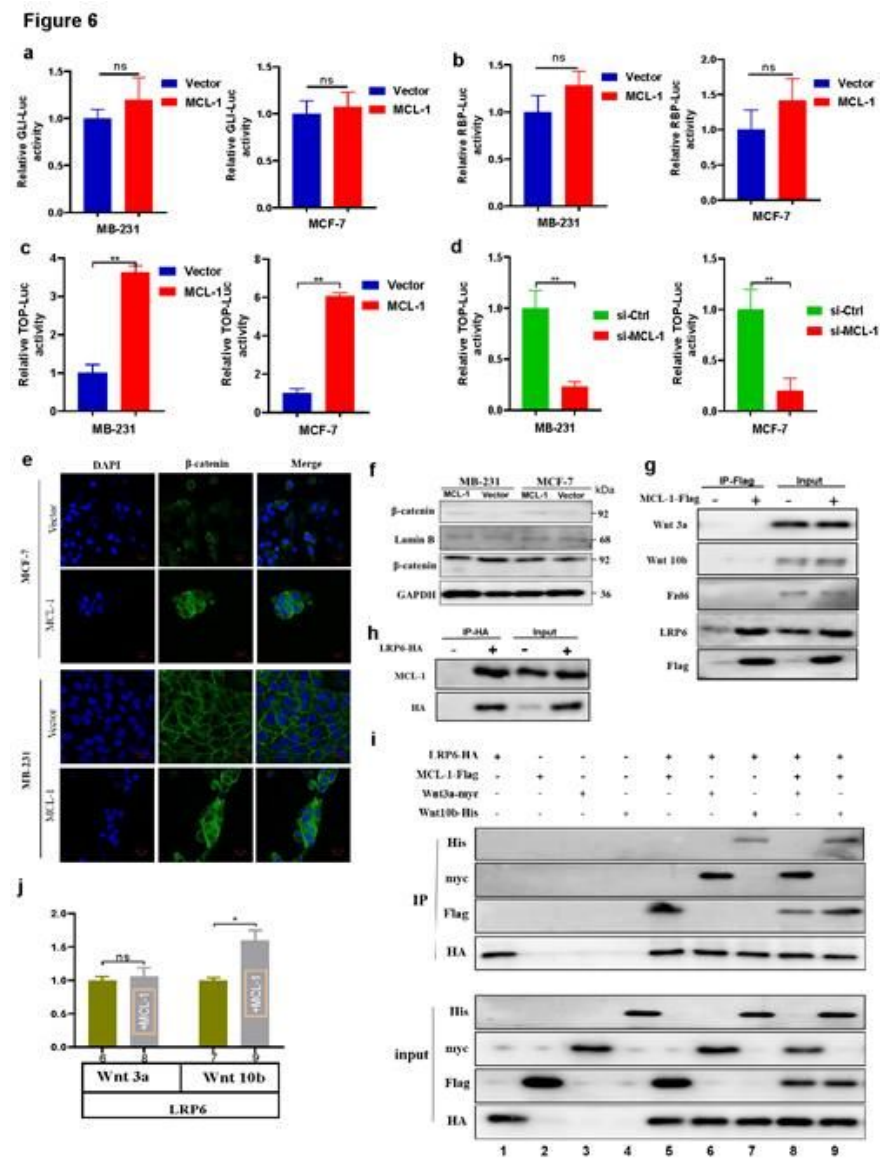

Figure 6

MCL-1 activates the WNT/ $\beta$-catenin pathway. (a-c) Luciferase reporter gene assay of MCF-7 and MB-231 cells transfected with MCL-1 plasmid or vector. The results shown are the mean \pm SD of the relative firefly/Renilla ratio. (d) Luciferase reporter gene assay of MCF-7 and MB-231 cells transfected with si-MCL-1 or si-Ctrl. (e) Representative images of the distribution of $\beta$-catenin in MCF-7 and MB-231 cells transfected with vector or MCL-1 plasmid by confocal. Scale bars: $20 \mu \mathrm{m}$. (f) The expression of $\beta$-catenin from nuclear and whole cell lysates of MCL-1 overexpression cell lines and vector cells were detected by western blot. (g) Co-IP assay between MCL-1 and WNT components (ligand: Wnt3a or Wnt10b; receptor: Fzd6 or LRP6). MB-231 cells were transfected with MCL-1-Flag or a control vector. The input on the right panel shows the levels of transfected Flag-MCL-1 and endogenous WNT components in Flag-tagged MCL-1 or vector control. (h) Co-IP assay between LRP6 and MCL-1. (i) MCL-1 enhanced the binding between Wnt10b and LRP6. HA-tagged LRP6-expressing cells were cocultured with Flag-tagged MCL-1, myc-tagged Wnt3a and His-tagged Wnt10b cells. The HA-tagged LRP6 was immunoprecipitated in this experiment. (j) Densitometric analysis showed the relative amounts of precipitated WNT ligand Wnt10b interacted with LRP6 affected by MCL-1. Those graphs and images are typical results of 3 repeat experiments (mean $\pm S D$, Students` $t$-test, *P®0.05, **P囚0.01). 
Figure 7
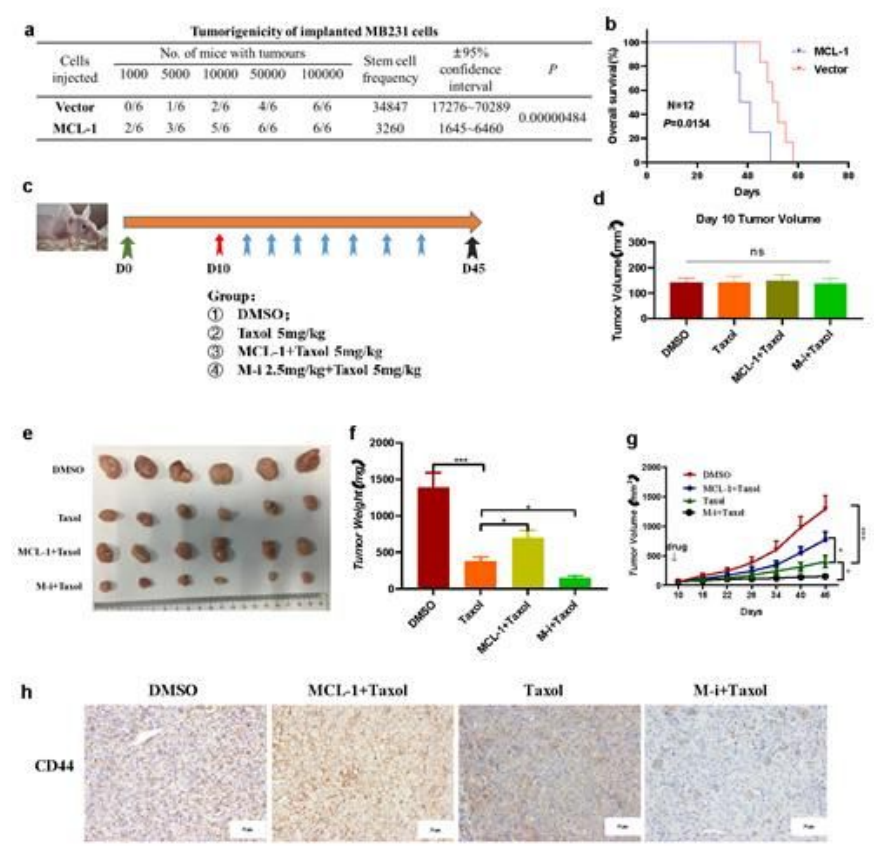

\section{Figure 7}

MCL-1 overexpression is associated with tumorigenesis and Taxol-resistance in vivo. (a) Different numbers MB231cells with or without MCL-1 overexpression are subcutaneously injected $(1000,5000,10000,50000,100000$ cells per mouse) into 6 Balc/c nude mice at 6 -week-old. The stem cell frequency is analyzed. (b) Overall survival is performed by Kaplan-Meier survival ( $\mathrm{n}=12$ mice $\mathrm{P}=0.0154)$. (c-g) 18 nude mice are subcutaneously injected with $1 \times 106 \mathrm{MB} 231$-vetector and 6 nude mice are with MB231-MCL-1 cells. After 10 days, the volume of subcutaneous tumor formation is about 150 mm3 and 18 nude mice would assign randomly to 3 groups. And then we intervene the mice by Taxol with or without M-i. We measure the tumor volumes every 3 days. (e-h) Image of mice xenograft tumors and CD44 ICH staining of tumor are shown. Those images are typical results (mean $\pm S D, * P \otimes 0.05, * \star * P \otimes 0.001)$.

Figure 8

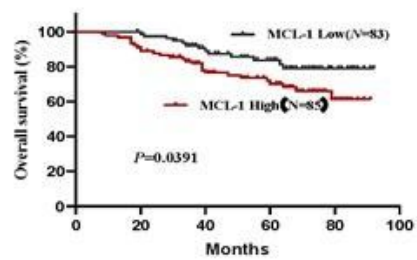

b
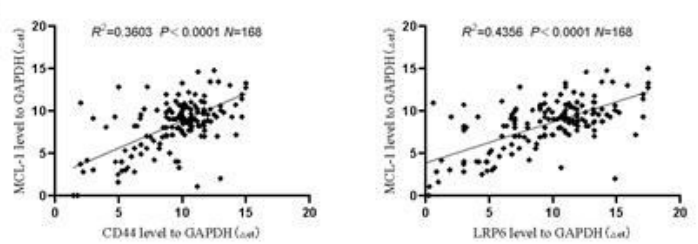

\section{Figure 8}

MCL-1 correlates with prognosis and cancer stem cell relevant genes expression. (a) We use a log-rank test to calculate the different between high (ICH score $=2$ or 3 ) of MCL-1 expression and low (ICH score $=0$ or 1 ). The result of Kaplan-meier plot of OS from 168 breast cancer patients is shown ( $\mathrm{P}=0.0391)$. (b) Pearson`s correlation analysis indicated a significant positive correlation between MCL-1 mRNA and stem cell relevant genes mRNA: CD44 (R2=0.3603, PQ $0.0001)$ and LRP6 (R2=0.4356, Pه0.0001) in 168 breast cancer biopsy tissues. 
Figure 9

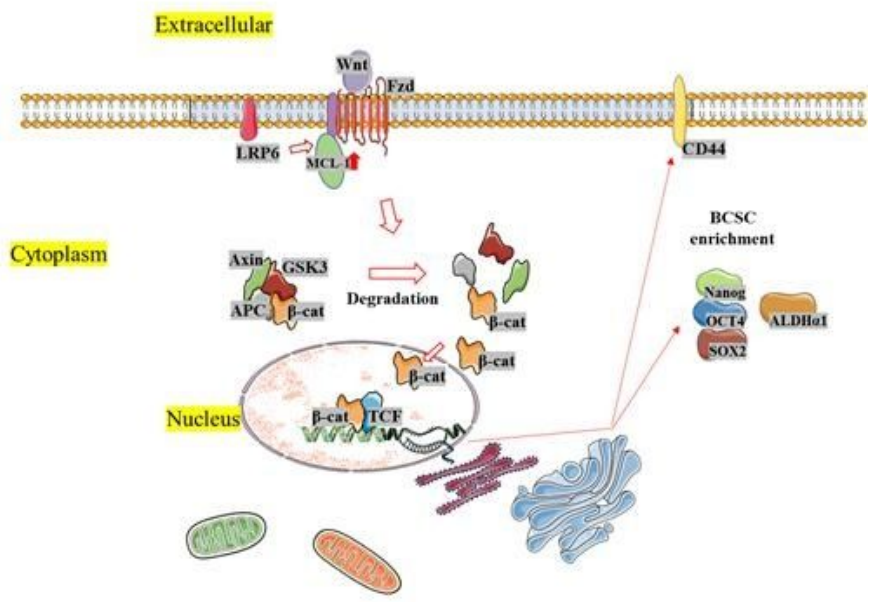

\section{Figure 9}

Our proposed model of WNT signal pathways that MCL-1 confers oncogenic role in breast cancer through modulating stemness property.

\section{Supplementary Files}

This is a list of supplementary files associated with this preprint. Click to download.

- SupplementarydataExcel1.xls 